\title{
TELEMONITORING OF USERS WITH PACEMAKERS: INFORMAL CAREGIVERS AT 5 YEARS AFTER IMPLANT
}

\author{
Antonio Lopez-Villegas ${ }^{1}$, Remedios Lopez-Liria, Cesar Leal-Costa and Daniel Catalan-Matamoros \\ Hospital of Poniente, University of Almeria, University of Murcia \& University Carlos III of Madrid, Spain
}

\begin{abstract}
Introduction: The use of telemonitoring of pacemakers is limited. Few studies have focused on their informal caregivers at long-term. To evaluate the burden borne to informal caregivers of patients with pacemakers, five years after implantation.

Methods: This is a controlled, non-randomised clinical trial. Data on baseline characteristics, workload and time worked were collected from informal caregivers, five years after implantation of pacemakers. Besides, the Spanish version of the Survey on Disabilities, Personal Autonomy, and Dependency Situations was used to get information on Clinical and social characteristics, levels of professionalism, duration and types of care, difficulties in providing care, health status, economic and job aspects, family and leisure.

Results: Fifty five patients with pacemakers finished the study. Of which, 50 needed to be helped by a caregiver, 18 were included in the telemonitoring and 32 in the hospital monitoring group. Overall, females represented $96.0 \%$ of the informal caregivers $(88.89 \%$ in telemonitoring and $100.0 \%$ in hospital monitoring group). The mean ages were $63.17 \pm 15.92$ and $63.13 \pm 14.56$ years, respectively $(p=0.83)$ in the groups of telemonitoring and hospital monitoring group. The majority $(88.0 \%)$ of the caregivers declared that they had to provide their services between 6 and 7 days per week $(83.33 \%$ in telemonitoring group.

Conclusions: The results confirm that there were no significant differences between the informal caregivers regarding to baseline characteristics, workload and time worked in both groups of follow-up.
\end{abstract}

\section{KEYWORDS}

Disease Burden, Informal Caregiving, Pacemaker Follow-Up, Remote Monitoring, Telemedicine

\section{INTRODUCTION}

The use of telemonitoring (TM) of pacemakers is limited. Few studies have focused on their informal caregivers at long-term.

\section{OBJECTIVE}

To evaluate the burden borne to informal caregivers of patients with pacemakers, five years after implantation.

\section{METHODS}

This is a controlled, non-randomised clinical trial. Data on baseline characteristics, workload and time worked were collected from informal caregivers, five years after implantation of pacemakers. Besides, the Spanish version of the Survey on Disabilities, Personal Autonomy, and Dependency Situations was used to get information on 1) Clinical and social characteristics; 2) Levels of professionalism, 3) Duration and types

${ }^{1}$ Contact Details Antonio Lopez Villegas: antoniolopezvillegas@andaluciajunta.es 
of care; 4) Difficulties in providing care, 5) Health status, 6) Economic and job aspects, 7) Family and leisure.

Patients were included in the study if: 1) they had been implanted with a single [VVI-VVIR] or double chamber [VDD-DDD] Medtronic Carelink® pacemakers, 2) they were over 18 years old, 3) they were capable of understanding and correctly performing the home auto-monitoring or had a caregiver and 4) they agreed to participate in the study by signing the informed consent. Patients were excluded if: 1) they were enrolled in another study, 2) they had any other cardiac device, 3) they refused to participate.

The Almeria Health Research Ethical Committee approved the trial protocol and the study was developed in accordance with the precepts of the Declaration of Helsinki and Spanish laws on data protection and patient rights. All patients signed the corresponding informed consent prior to their enrolment, and appropriate measures were taken to ensure data privacy. The trial protocol was registered in ClinicalTrials.gov (Identifier: NCT02234245).

\section{RESULTS}

Fifty five patients with pacemakers finished the study. Of which, 50 needed to be helped by a caregiver, 18 were included in the TM and 32 in the hospital monitoring group (HM). Overall, females represented $96.0 \%$ of the informal caregivers (88.89\% in TM and $100.0 \%$ in HM group). The mean ages were $63.17 \pm 15.92$ and $63.13 \pm 14.56$ years, respectively $(p=0.83)$ in the groups of TM and HM group. The majority $(88.0 \%)$ of the caregivers declared that they had to provide their services between 6 and 7 days per week $(83.33 \%$ in TM versus $90.63 \%$ in HM group). No significant differences between the two groups were found in any of questions of the Survey administrated.

\section{CONCLUSIONS}

The results confirm that there were no significant differences between the informal caregivers regarding to baseline characteristics, workload and time worked in both groups of follow-up.

\section{ACKNOWLEDGEMENT}

The study has been financially supported by the General Secretariat for Research, Development and Innovation, Regional Government of Andalusia (Spain), project reference number PI/0256/2017, under the research call "Development and Innovation Projects in the Field of Biomedicine and Health Sciences," 2017.

\section{REFERENCES}

López-Villegas A, Catalán-Matamoros D, Robles-Musso E, Peiró S. Workload, time and costs of the informal cares in patients with tele-monitoring of pacemakers: the PONIENTE study. Clin Res Cardiol. 2016;105(4):307-13. doi: 10.1007/s00392-015-0921-5. Epub 2015 Sep 30. PMID:26423396

Instituto Nacional de Estadística. Encuesta sobre Discapacidades, Autonomía personal y situaciones de Dependencia. 2008. http://www.ine.es/daco/daco42/discapa/edad_cui.pdf 